\title{
EVALUACIÓN QUÍMICO PROXIMAL Y FÍSICO QUÍMICA DE 5 ESPECIES Y/O VARIEDADES DE AJÍES (CAPSI- CUM) IDENTIFICADOS EN LA PROVINCIA DE OXAPAMPA, REGIÓN PASCO*
}

\author{
Gamarra Mendoza, Norma ${ }^{1}$
}

Facultad de Ingeniería en Industrias Alimentarias de la Universidad Nacional del Centro

\begin{abstract}
RESUMEN
Se realizó la colecta de 5 especies y variedades de ajíes (Capsicum) en la provincia de 0xapampa, los cuales fueron evaluados de acuerdo a sus características morfofenotípicas, y seguidamente se les asignó el nombre científico, tipo, variedad y nombre común. Estas especies fueron cultivadas en invernaderos a fin de uniformizar las condiciones de cultivo. Los frutos cosechados fueron evaluados físicamente: peso, volumen, longitud, diámetro transversal y forma, características que sirven como indicadores de madurez. Asimismo se determinó el valor de $\mathrm{pH}$, porcentaje de acidez (\%ácido cítrico), sólidos solubles y relación de madurez cuando los frutos presentaron un color rojo intenso. El C. chínense Hab. fatalii red. 4PAL ISC014 comparado al C, chínense Charapita 4 PAL ISC0 10 presento una mayor relación de madurez $(57,73)$ y $(20,15)$, respectivamente. Esto podría deberse a la diferencia del contenido de sólidos solubles y ácidos orgánicos. Respecto a la composición química, el $C$. frutescens, presento el mayor contenido de materia seca (42,14\%), y se ve reflejado en el mayor contenido de proteínas $(6,42 \%)$, fibra $(6,22 \%)$ y carbohidratos $(26,58 \%)$ a diferencia de las otras especies que muestran un menor porcentaje de estos componentes.
\end{abstract}

Palabras clave: ajíes, capsicum: annuum, baccatum, chínense, frutescens, pubecens

\begin{abstract}
Collection was performed 5 species and varieties of peppers (Capsicum) in the province of Oxapampa, which were evaluated according to their characteristics morfofenotípicas, and then were assigned the scientific name, type, variety and common name. These species were grown in greenhouses in order to standardize the culture conditions. The harvested fruits were assessed physically: weight, volume, length, transverse diameter and shape, features which serve as indicators of maturity. It also determined the $\mathrm{pH}$, acidity percentage (\% citric acid), soluble solids and relationship maturity when fruits showed an intense red color. The $C$. chinense Hab.fatalii network. 4PAL ISC014 compared to $C$, chinense charapita 4 PAL ISCO 10 shows a higher ratio of maturity (57.73) and (20.15), respectively. This could be due to the difference in soluble solids and organic acids. Regarding the chemical composition, the $C$. frutescens, showed the highest content of dry matter (42.14\%), and is reflected in the higher protein content $(6.42 \%)$, fiber $(6.22 \%)$ and carbohydrate $(26.58 \%)$ unlike other species which show a lower percentage of these components.
\end{abstract}

Key words: pepper, capsicum: annuum, baccatum, chínense, frutescens

\footnotetext{
* Este trabajo de investigación fue recibido el 20/06/2009 retornado para su revisión 26/10/2009 y aprobado para su publicación 16/11/2009

1Email: ngamarra@uncp.edu.pe
} 


\section{INTRODUCCIÓN}

El género Capsicum, comúnmente denominado aji en el Perú, juega un rol importante en el sector hortícola del país. El Consejo Internacional de Recursos Fitogenéticos con sede en Costa Rica (1979), reconoció a nivel mundial la importancia socioeconómica y científica de este género, considerado dentro de los recursos fitogenéticos de primera prioridad (Ortiz,1983). El género Capsicum presenta una gran oferta de especies y variedades, los cuales reúnen características de precocidad, productividad y demanda comercial que los convierte en un recurso genético promisorio para Ios sistemas productivos y hace viable su inclusión en agrocadenas de los mercados nacional e internacional. Weiss (2002) indica que la producción total y mundial de ajíes para el año 2000 alcanzó de $14-15$ millones de toneladas. El ají por su contenido de componentes bioactivos (capsacinoides, carotenoides, compuestos fenólicos, ácidos, etc.) de interés medicinal, farmacológico y alimentario cobra importancia para su comercialización, investigación y mejoramiento de su producción. El potencial de compuestos naturales que ofrece el género Capsicum es impresionante, sin embargo en nuestro país muy poco se ha investigado para utilizar estas ventajas comparativas y competitivas, ya que las tendencias actuales y a nivel mundial, consideran prioritarias la búsqueda de nuevos compuestos bioactivos, ingredientes, sabores y aromas naturales de matrices vegetales, para utilizarlos como alimentos saludables y nutritivos que orienten a mejores oportunidades de comercialización para posesionarse en mercados verdes de alimentos funcionales, nutracéuticos, cosmecéuticos, productos orgánicos e insumos exclusivos. Además de estos usos, los frutos se utilizan -procesados 0 en fresco y bajo diferentes formas- para la preparación y aliño de los alimentos. La calidad de los frutos del ají y de sus subproductos depende del color, el aroma y la pungencia (Kirschbaum et al., 2002a; y Macrae et al., 1993); particularmente el color rojo y amarillo del Capsicum, originado por la presencia de pigmentos carotenoides, que lo hacen importante en la industria de especias, siendo la especie $C$. annuum $\mathrm{L}$. la de mayor distribución. El Perú es uno de los centros de origen de la mayor cantidad de especies de Capsicum, que data desde la época del incanato, esta diversidad biológica de nuestro país nos exige asumir una responsabilidad en el uso sostenible y conservación de estos recursos y la necesidad de prepararnos y exigirnos para aprovechar las nuevas tendencias del mercado.

En este artículo, se describe la composición química y características fisicoquímicas de las especies de ajíes colectadas y cultivadas en la provincia de 0xapampa - Región Pasco. Investigación realizada gracias al cofinanciamiento entre la UNCP - INCAGRO.

\section{MATERIALES Y MÉTODOS}

\section{Lugar de ejecución}

El trabajo de investigación se desarrollo en:

- Laboratorio de Química de Alimentos de la Facultad de Industrias Alimentarias.

- Herbario del Departamento de Biología de la Universidad Nacional Agraria La Molina.

- Parcelas Experimentales de Cultivo de La Sub Gerencia Regional Oxapampa, Región Pasco.

- Escuela de Formación Profesional de Agronomía - Oxapampa. Universidad Nacional Daniel Alcides Carrión.

- Laboratorio del Análisis de Alimentos del Instituto Tecnológico Santiago Antúnez de Mayolo - PalianHuancayo.

\section{Material biológico}

Género Capsicum, cultivables y no cultivables de la Provincia de 0xapampa

\section{Materiales, Instrumentos y Equipos}

Equipo Kjeldalh, Equipo Soxhlet, Balanza analítica, pHmetro 0-14, Incubadora de $0-60^{\circ} \mathrm{C}$, Vortex, Agitador Magneto, Batidora - Homogenizadora, Horno de Calcinación, Esterilizadora, Equipo de Titulación. Matraces de 125, 250, 500 y $1000 \mathrm{~mL}$; Probetas de 100, 250, 500 y $1000 \mathrm{~mL}$; Fiolas de 100, 250, 500 y 1000 $\mathrm{mL}$; Vasos de precipitados 100, 250, 500 y $1000 \mathrm{~mL}$; Bandejas y Recipientes de $5 \mathrm{~kg}$; Pipetas de 1,5 y 10 $\mathrm{mL}$; Micropipetas de 50 - 1000 ul; Termómetro de 10 $-150^{\circ} \mathrm{C}$, refractómetro $0-32^{\circ} \mathrm{Bx}$.

\section{Insumos y Reactivos}

Reactivos para determinación de composición química proximal; ácido clorhídrico p.a, catalizadores inorgánicos, hexano p.a; ácido acético glacial; ácido sulfúrico Q.P; fenolftaleína al $1 \%$; alcohol de $96 \%$; hidróxido de sodio p.a. 


\section{MÉTODO}

\section{Análisis Fisicoquímico}

- Contenido de sólidos solubles, método del refractómetro, Mendez et al., (2004).

- Determinación de pH, Mendez et al., (2004).

- Determinación de acidez total, método volumétrico, Mendez et al., (2004).

\section{Análisis Químico proximal:}

- Determinación de Proteína

Se realizó, FAO (2004).

- Determinación de Fibra

Se realizó, FAO (2004).

- Determinación de Carbohidratos

Se realizó, Según FAO 2004.

- Determinación de grasa

Se realizó Según FAO 2004.

- Determinaron de ceniza

Se realizó, Según FAO 2004.

- Determinación de Humedad

Se realizó, Según FAO 2004.

\section{RESULTADOS}

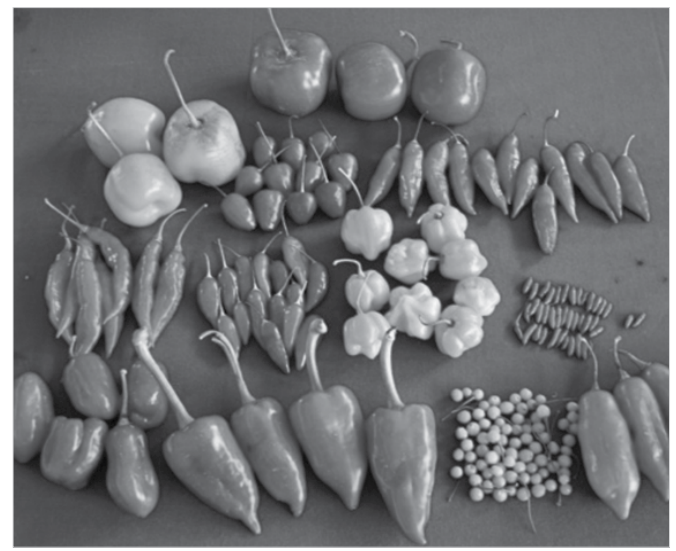

Figura 1. Especies de Capsicum colectadas en la provincia de Oxapampa, región Pasco.

\section{Análisis fisicoquímico}

Cuadro 1. Características fisicoquímicas del género Capsicum obtenidas en la provincia de 0xapampa.

\begin{tabular}{|c|c|c|c|c|}
\hline $\begin{array}{c}\text { Especies y/o } \\
\text { Variedades de } \\
\text { Capsicum }\end{array}$ & PH & $\begin{array}{c}\text { Sólidos } \\
\text { Solubles } \\
(\%)\end{array}$ & $\begin{array}{c}\text { Acidez } \\
\text { Titulable } \\
\text { (\% Ac. Cítrico) }\end{array}$ & $\begin{array}{l}\text { Índice de madurez } \\
\text { (Relación de } \\
\text { SS/\%acidez total) }\end{array}$ \\
\hline $\begin{array}{l}\text { C. pubescens } \\
\text { Rocoto } \\
2 \text { HUAN ANC } 02\end{array}$ & 5.08 & 5,2 & 0.176 & 29,00 \\
\hline $\begin{array}{l}\text { C. chínense } \\
\text { Hab. Scotch bon } \\
\text { 4PAL.BUE.AIR.5 }\end{array}$ & 5,08 & 6,13 & 0,21 & 29,21 \\
\hline $\begin{array}{l}\text { C. chínense } \\
\text { Hab.fatalii red v. } \\
\text { 4PAL ISCO14 }\end{array}$ & 5,99 & 6,93 & 0,12 & 57,73 \\
\hline $\begin{array}{l}\text { C, chínense } \\
\text { Charapita } \\
4 \text { PAL ISCO } 10\end{array}$ & 4,72 & 9,06 & 0,45 & 20,15 \\
\hline $\begin{array}{l}\text { C. baccatum } \\
\text { Ají omnicolor } \\
\text { 4PAL.ISCO } 7\end{array}$ & 5,23 & 10,0 & 0,31 & 32,25 \\
\hline $\begin{array}{l}\text { C .baccatum } \\
\text { Peruvian pointer } \\
4 \text { PAL.MAY.3 }\end{array}$ & 5,08 & 8,4 & 0,36 & 23,33 \\
\hline $\begin{array}{l}\text { C.baccatum } \\
\text { Inca Red Drop } \\
5 \text { PUE. BERM.2 }\end{array}$ & 4,86 & 8,4 & 0,29 & 28,96 \\
\hline $\begin{array}{l}\text { C.annuum } \\
\text { Paprika } \\
3 \text { POZUZO } 1\end{array}$ & 4,82 & 9,0 & 0,23 & 38,69 \\
\hline $\begin{array}{l}\text { C.frutescens } \\
\text { Aji chuncho } \\
\text { 4 PAL.RAYA } 1\end{array}$ & 4,62 & 9,0 & 0,44 & 20,30 \\
\hline
\end{tabular}




\section{Análisis químico proximal}

Cuadro 2. Composición químico proximal del género Capsicum obtenido de la provincia de 0xapampa

\begin{tabular}{|c|c|c|c|c|c|c|c|c|c|}
\hline Componentes & $\begin{array}{l}\text { C.pubescens } \\
\text { "Rocoto" } \\
2 \text { HUAN ANC } 02\end{array}$ & $\begin{array}{c}\text { C.chinense } \\
\text { "Habanero } \\
\text { Fatalii Red } \\
\text { vel aff" } \\
4 \text { PAL ISCO } \\
14\end{array}$ & $\begin{array}{l}\text { C.chinense } \\
\text { "Habanero } \\
\text { Scotch Bonnet } \\
\text { Orange" } \\
\text { 4 PAL BUE } \\
\text { AIR 5 }\end{array}$ & $\begin{array}{l}\text { C.chinense } \\
\text { "Charapita" } \\
4 \text { PAL ISCO } 10\end{array}$ & $\begin{array}{l}\text { C.baccatum } \\
\text { "Aji Omnicolor" } \\
4 \text { PAL. ISCO } 15\end{array}$ & $\begin{array}{c}\text { C.baccatum } \\
\text { "Peruvia } \\
\text { pointer" } \\
\text { 4 PAL MAY } \\
03\end{array}$ & $\begin{array}{c}\text { C.baccatum } \\
\text { "Inca Red } \\
\text { Drop" } \\
5 \text { PUE } \\
\text { BERM } 2\end{array}$ & $\begin{array}{l}\text { C. frutescens } \\
\text { "Ají chuncho" } \\
4 \text { PAL RAY. } 1\end{array}$ & $\begin{array}{l}\text { C. annuum } \\
\text { "Paprika" } \\
3 \text { POZUZO } 1\end{array}$ \\
\hline Humedad (\%) & 90.71 & 86 & 90 & 78,4 & 90,26 & 88,88 & 88,17 & 57,86 & 89,42 \\
\hline Materia seca (\%) & 9.29 & 14,0 & 10,0 & 21,6 & 14,64 & 11,11 & 11,82 & 42,14 & 10,58 \\
\hline Ceniza (\%) & 0.52 & 0,89 & 0,74 & 1,13 & 0,91 & 1,63 & 0,74 & 1,85 & 0,66 \\
\hline Grasa $(\%)$ & 0.32 & 0,59 & 0,38 & 0,95 & 2,18 & 0,9 & 2,79 & 1,07 & 0,30 \\
\hline Proteína (\%) & 0.99 & 0,78 & 0,96 & 2,6 & 1.51 & 1.28 & 1.23 & 6,42 & 1,61 \\
\hline Fibra cruda (\%) & 1.65 & 4,9 & 4,29 & 6,5 & 1.81 & 2,11 & 2,23 & 6,22 & 2,07 \\
\hline Carbohidratos (\%) & 5.82 & 6,83 & 3,36 & 10,42 & 4.84 & 5.20 & 4.84 & 26,58 & 5,96 \\
\hline
\end{tabular}

\section{DISCUSIÓN}

En la figura 1 y 2, se muestra los lugares de colección y las especies de Capsicum colectadas de la provincia de Oxapampa, Región Pasco. Los cuales fueron evaluadas morfológicamente para agruparlas dentro de las especies que les corresponde según el IPGRI. En el cuadro 1, se muestra los resultados de valores de $\mathrm{pH}$, sólidos solubles, acidez total (\% de ácido cítrico) y relación de madurez (\% de sólidos solubles/\%Acidez Total) de las distintas especies de Capsicum. El rango de pH de las especies en estudio se encuentra entre 4,62 a 5,99 comparado con los resultados de $\mathrm{pH}(3,0$ a 5,4$)$ de 11 cultivares de chilli habanero (Pino et al, 2006) los resultados son muy similares. Respecto, al contenido de acidez total expresado en porcentaje de ácido cítrico se obtuvo en un rango de 0,12 a $0,45 \%$, estudios realizados en diferentes solanáceas señalan que la cantidad de acidez debe tener una tendencia decreciente siendo un patrón característico conforme avanza el proceso de maduración (Méndez, et al. 2004), esta disminución es debida a la degradación oxidativa del almidón, azúcares y ácidos orgánicos que se acumulan en la etapa de alta división celular para su utilización en la síntesis de nuevas proteínas y ARNm, así como de nuevos pigmentos y componentes de sabor, procesos anabólicos que requieren de energía y esqueletos carbonados, los cuales son proporcionados mediante la respiración (Seymour, 1993).
La solubilidad de minerales, la absorción de agua, nutrimentos y gases, así como varios procesos de difusión que ocurren dentro de la planta, dependen también de la temperatura (Ortiz, 1987), de esta manera estos cambios no solo afectan al crecimiento sino también a todo el proceso metabólico de síntesis y acumulación de diversos compuestos responsables de los cambios estructurales, coloración, pungencia y características organolépticas. Los vegetales contienen pequeñas cantidades de ácidos orgánicos que actúan como intermediarios metabólicos (ejemplo el ciclo de los ácidos tricarboxílicos, el ciclo del glioxilato, o la ruta del acido shiquimico) que se pueden acumular en forma de vacuolas. En cuanto al contenido de sólidos solubles de las especies en estudio se encuentran entre 5,2 y 9,06 Brix, comparado con los sólidos solubles (6,4 a 9,1 ${ }^{\circ}$ Brix) de cultivares de chilli habanero (Pino et al.,2006) podemos observar que los resultados son muy cercanos, excepto el $C$. pubescens rocoto 2 HUAN ANC $02 y$ el C. chínense Hab. Scotch bon 4PAL.BUE.AIR.5, muestran una menor cantidad de sólidos solubles que podría deberse a sus características intrínsecas.

En el cuadro 2, se observa la composición química de las 5 especies de ajíes, el $C$. pubescens, muestra el mayor porcentaje de humedad, el espesor del tejido es de 2 a 2,5 mm y la placenta es de mayor espesor de aproximadamente de $3 \mathrm{~mm}$ y muy poroso el cual alberga un alto porcentaje de humedad. Sin embargo el $C$. frutescens muestra un menor porcentaje de humedad, el espesor del tejido es de $0,5 \mathrm{~mm}$, tiene poca 
cantidad de tejido en relación al contenido de semillas, su contenido de materia seca es alto comparado a las otras especies, esto se ve reflejado en el contenido de fibra, carbohidratos y proteínas; el menor contenido de proteínas muestran las especies de $C$. pubescens, $C$. chinense 4 PAL ISC0 14 y 4 PAL. BUE. AIR 5. Este bajo contenido de proteínas puede deberse a que los frutos almacenan principalmente ácidos orgánicos y carbohidratos (Wills, 1998) en un rango de 4.07 $5.82 \%$ (fruto fresco libre de semillas).

Respecto al contenido de cenizas de las muestras en estudio se encuentran entre 0,52 a 1,85\%. Los de chilli habanero contienen de 0,5 a 1,2\% se observa que los resultados de contenido de ceniza de las muestras en estudio se aproximan con variaciones muy ínfimas, excepto el $C$. baccatum 4 PAL MAY 3 y $C$. frutescens 4 PAL RAY. 1 cuyo contenido de ceniza se encuentra por encima del límite superior al de chilli habanero.

Teniendo en cuenta que el contenido de aceite en Capsicum es < 8\%; en las muestras de Capsicum pubescens y $C$. annuum el contenido de grasa es menor y son características propias de estos frutos, pues en casi todas las frutas y hortalizas frescas los lípidos representan menos del $1 \%$ (Fennema, 2000), esa cantidad comparado con la composición de otras fuentes, se encuentra cerca al limite inferior, es posible que las estructuras que contienen a estos compuestos consisten en una matriz de proteínas y lípidos, además los lípidos pueden acumularse en las paredes celulares.

\section{CONCLUSIONES}

Las características fisicoquímicas de $\mathrm{pH}$, porcentaje de acidez total y sólidos solubles determinadas en las diferentes especies de Capsicum, presentan valores característicos de cada especie que también son indicadores de madurez, ya que conforme alcanzan este estadio disminuye el porcentaje de acidez y aumenta el valor de $\mathrm{pH}$. Respecto a la composición químico proximal, la especie que presento mayor contenido de materia seca, proteínas, fibra y carbohidratos a diferencia de las otras especies es el $C$. frutescens, siendo un fruto muy pequeño y con poca cantidad de pericarpio en relación al contenido de semillas, asimismo contiene un bajo porcentaje de humedad.

\section{LITERATURA CITADA}

Azcón-Bieto, J. y Talon, M. 1993. Bioquímica y fisiología vegetal. México: Interamericana McGraw Hill.

Fennema, 0. 2000. Quimica de los alimentos (2 ed.). Zaragoza, España: Acribia S.A.

IPGRI. 1995. Genetic resources of capsicum. IPGRI Secretariat. Rome, Italy. En: www.ipgri.cgiar.org.

Macrae, R., Robinson, R. y Sadler, M. 1993. Encyclopaedia of food science food technology and nutrition. Academic Press, Nueva York. pp. 3496-3504.

Mendez, M., Ligarreto, G., y Hernandez, S. M. 2004. Evaluacion del crecimiento y determinación de índices de cosecha en frutos de cuatro materiales de aji (Capsicum sp.) cultivados en la amazonia Colombiana. Agronomia Colombiana , 22 (1), 7 - 17.

Ortiz, R. 1983, Utilización de descriptores en la caracterización de líneas de Capsicum. UNA

Ortiz, C. 1987. Elementos de agroclimatología cuantitativa. Chapingo: UACH.

Seymour, G., Taylor, J., y Tucker, G. 1993. Biochemistry of fruit ripening. London: Chapman \& hall

Weiss, E.A. 2002. World Production and Trade. CABI Publishing, CAB International, Wallingford, UK.

Wills, R., McGlasson, D., y Graham, D. 1998. Postharvest. An introduction to the physiology and handling of fruit, vegetables and ornamental. (4th ed.). 